\title{
Surgical management of papillary thyroid cancer: review of current evidence and consensus
}

\author{
Umesh Jayarajah $^{1}$, Kavinda Nagodavithane ${ }^{1}$, Oshan Basnayake ${ }^{1}$, Sanjeewa Seneviratne \\ ${ }^{1}$ Professorial Surgical Unit, National Hospital of Sri Lanka, Colombo, Sri Lanka \\ ${ }^{2}$ Department of Surgery, Faculty of Medicine, University of Colombo, Sri Lanka
}

Keywords: Papillary thyroid cancer; surgical management; prophylactic lymph node dissection

\begin{abstract}
The optimal surgical management of papillary thyroid carcinoma has been an ongoing debate. Most recommendations in clinical practice guidelines are based on large retrospective studies and expert opinion. The objective of the article is to summarize the recent evidence and main arguments related to the surgical management of papillary thyroid carcinoma. A definitive correlation between locoregional recurrence and long-term survival and the extent of thyroid resection or lymph node dissection have not been established through randomized controlled clinical trials. Due to the low rates of recurrence and mortality associated with papillary thyroid cancer, large scale prospective randomized controlled trials that will help identify the optimal surgical management are unlikely to be available in the future as well. According to current consensus, hemithyroidectomy is sufficient for low-risk disease whereas total thyroidectomy should be performed in those with high-risk features. The place of therapeutic and prophylactic central compartment and lateral neck dissection is discussed based on evidence on short-term and long term outcomes. Furthermore, postoperative staging and dynamic risk stratification are important in determining adjuvant therapy and a follow-up plan.
\end{abstract}

\section{Introduction}

Thyroid cancer is common in both developing and developed countries and is one of the most rapidly increasing cancers in many countries including the USA and UK (1). Papillary thyroid carcinoma (PTC) is the commonest type of primary thyroid malignancy (2). The rapid increase in incidence in most parts of the world has been largely attributed to the increased diagnosis of asymptomatic or subclinical lesions, although an actual increase is also a likely contributor $(2,3)$.

\footnotetext{
Correspondence: Sanjeewa Seneviratne

E-mail: sanjeewa@srg.cmb.ac.lk

Received: 05-09-2019 Accepted: 12-07-2019

(iD) http://orcid.org/0000-0003-0310-1387
}

DOI: http://doi.org/10.4038/sljs.v37i4.8617
The debate on optimal surgical treatment for PTC has been gaining popularity, especially in terms of the need for surgery and the extent of thyroid resection for low-risk disease as well as the need and the extent of neck lymph node dissection. The main objectives in managing PTC are reducing diseaserelated morbidity, disease recurrence, and morbidity of treatment while improving disease-specific and overall survival. Surgical management of PTC is mainly carried out with therapeutic intentions but is also considered in situations of diagnostic uncertainty. The extent of gland removal, intraoperative techniques for preservation of related nerves and parathyroid glands are important considerations of surgical management. Due to the low recurrence and diseasespecific mortality rates from PTC, to gain sufficiently powered level 1 evidence on the ideal initial surgical management requires a large-scale, expensive and prolonged randomized control trial which is impractical (4). Therefore, management protocols are primarily based on large scale retrospective analyses and expert opinion.

\section{Hemithyroidectomy versus total thyroidectomy}

Optimal surgical management is the cornerstone of treatment which improves prognosis while thyroid-stimulating hormone (TSH) suppression and radioiodine ablation act as adjuncts to the treatment. Recent studies have attempted to resolve the ongoing argument regarding the place of total thyroidectomy versus hemithyroidectomy concerning the impact on outcomes. Total thyroidectomy has the potential benefits of removing possible multifocal disease involving the contralateral lobe, ability to administer radioactive iodine therapy to ablate residual thyroid including metastatic disease and follow up for tumour recurrence with serum thyroglobulin levels.

Hemi thyroidectomy is recommended in certain instances with diagnostic uncertainty such as in patients with Thy 3 (Bethesda IV) fine-needle aspiration cytology (5). Follicular variant of PTC may fall into Thy 3 f category when the nuclear features minimally favour PTC. Furthermore, when a patient presents with a well-defined small lesion suggestive of PTC, a hemithyroidectomy may be performed as a diagnostic procedure (6). 
The extent of the therapeutic surgery depends on the size of the primary tumour, status of the contralateral lobe, extrathyroidal spread (loco-regional or distant metastasis) and patient factors. When the size of the primary tumour is less than $10 \mathrm{~mm}$, it is defined as micropapillary carcinoma.

Unifocal micropapillary carcinoma without evidence of metastasis is managed with hemithyroidectomy alone although other associated high-risk factors may warrant total thyroidectomy (7). Extra thyroidal extension, multifocal disease, bilateral involvement, poor differentiation and infiltrative growth pattern are recognized as high-risk factors (8). In micropapillary carcinoma, the presence of multifocal disease involving both lobes will require a total thyroidectomy (7). Therefore, personalized decision making should be considered in patients with other above-mentioned risk factors. Management of PTC other than micropapillary category depends on the size of the tumour and other associated risk factors.

Lower risk of local recurrence is reported after total versus hemithyroidectomy in some studies possibly due to the propensity for multifocality in PTC $(9,10)$. However, with proper patient selection based on risk stratification, locoregional recurrence rates have been reported to be less than 1$4 \%$ (11). Evolution of the surgical management of PTC was largely based on retrospective data. Earlier, all PTC larger than $1 \mathrm{~cm}$ in size were managed with total thyroidectomy irrespective of the local, nodal or distant metastatic status (12). This practice was largely based on analysis of retrospective data from the national cancer database which included 52,173 patients who underwent thyroid surgery $(43,227$ had a total thyroidectomy, 8946 had hemithyroidectomy) between the period of 1985-1998 (13). This study showed that total thyroidectomy group had a low risk of 10 -year recurrence $(7.7 \%$ vs. $9.8 \%, \mathrm{P}<0.05)$ and mortality compared with hemithyroidectomy $(p=0.04$, $\mathrm{p}=0.009$, respectively). Since this was a retrospective analysis, the data in relation to the high-risk category such as extrathyroidal extension and extent of resection were not available. Because of these reasons, the decision to proceed with total or partial thyroidectomy remained unclear. Further analysis of the database was carried out by Adam et al (14) which included 61,775 patients who had a primary tumour size of $1-4 \mathrm{~cm}$ from the same database between 1998 and 2006. The complexity and severity of the disease were considered as additional variables. The overall survival benefit which was observed in the previous study was not demonstrated. A similar observation was made during subgroup analysis based on tumour size of $1-2 \mathrm{~cm}$ and $2-4 \mathrm{~cm}$ (14). Furthermore, 5432 PTC patients from Surveillance, Epidemiology, and End Results (SEER) program were analysed by Haigh et al. and found to have no difference between the total versus hemithyroidectomy groups in terms of 10-year overall survival (15). This analysis was carried out in relation to the risk category by AMES (Age, Metastases, Extent of disease, and Size) classification system. A recent analysis of the same database including 23,605 patients failed to show an overall survival difference between the two methods of thyroidectomy (16). Moreover, multivariate analyses including the stage of the disease, gender, radioiodine use failed to demonstrate a significant difference in overall survival in relation to the extent of surgery.

The paradigm shift from mandatory radioiodine ablation to selective use of radioiodine has also contributed to the selection with regard to the extent of surgery. Furthermore, the use of neck ultrasound scan and serial thyroglobulin measurements for the detection of recurrence than using radioiodine scan has also facilitated the decision-making process. However, the follow-up burden is a significant factor in hemithyroidectomy (17). The interpretation of thyroglobulin levels in the setting after a hemithyroidectomy is challenging than with total thyroidectomy and ultrasonographic detection of central nodal recurrence has also interfered after hemithyroidectomy.

\section{Minimizing surgical morbidity}

Intraoperative strategies to minimize the nerve damage and preservation of parathyroid glands are important to minimize postoperative morbidity following thyroidectomy. The two main nerves at risk are the external branch of the superior laryngeal nerve (EBSL nerve - supplies the cricothyroid muscle to alter the tension of vocal cords) and the recurrent laryngeal nerve (which supplies the all the other laryngeal muscles except cricothyroid muscle). The ideal way to prevent the damage to EBSL nerve is direct visualization and the preservation $(18,19)$. Meticulous skeletonisation of the superior pole while dissecting close to the thyroid capsule and ligation of superior thyroid vessels is practised to minimize nerve injury. In relation to recurrent laryngeal nerve, routine visualization of the nerve during dissection of the area at risk has shown a lower incidence of nerve damage than avoiding the nerve $(18,19)$. Intra-operative neuromonitoring is also used to prevent recurrent laryngeal nerve damage. However, a systematic review with meta-analysis comparing nerve visualization versus intra-operative neuromonitoring showed no statistical significance (20).

Preservation of parathyroid glands is also achieved by direct visualization. All measures should be undertaken to perform a meticulous dissection on the thyroid capsule and ligation of the inferior thyroid arterial branches close to the capsule of the gland $(21,22)$. Autotransplantation of parathyroid glands into the sternomastoid muscle should be considered in an event of inadvertent removal of the gland or in a situation where the 
adequacy of vascularity of the glands is in doubt. Routine inspection of the thyroidectomy specimen should be carried out to identify inadvertently removed parathyroid glands.

\section{Lymph node dissection in papillary thyroid cancer}

\section{Prophylactic central compartment lymph node dissection}

Retrospective analyses of large databases suggest that lymph node metastasis is associated with higher mortality particularly in older patients ( $>45$ years) (23). However, in those with clinical N0 disease, prophylactic central compartment node dissection (PCCND) has not proven to be beneficial in improving disease-specific or recurrence-free survival (5). PCCND is useful for accurate staging, reduction of locoregional recurrence, reduction of thyroglobulin concentration and in de-bulking before radioiodine therapy $(5,24)$. However, the loco-regional recurrence is reported to be only $2 \%$ in pN1 but clinically negative lymph nodes (cN0) and approximately $4 \%$ in patients with less than five involved lymph nodes (25). Therefore, to prevent one loco-regional recurrence, an estimated 20-30 PCCNDs are required (5).

Several systematic reviews and meta-analyses have been performed on the usefulness of PCCND. However, they contain mainly data from retrospective series. The findings were non-uniform and recent meta-analyses failed to detect significant differences in rates of loco-regional recurrences or permanent complications in those undergoing PCCND (2628). Therefore the British Thyroid Association guidelines do not recommend PCCND for those without clinical or radiological evidence of nodal involvement and those with low-risk factors $(<45$ years, classical papillary thyroid histology, unifocal disease, tumour diameter less than or equal to $4 \mathrm{~cm}$, and absence of extra thyroid extension). Bilateral PCCND may be offered individually in those with high-risk features (age $\geq 45$ years, unfavourable histology subtypes, multifocal disease, tumours $>4 \mathrm{~cm}$ in diameter and extension beyond the thyroid) after weighing the potential benefits against the morbidity due to surgery. Unilateral PCCND has no proven advantage and thus not recommended (5).

\section{Prophylactic lateral neck lymph node dissection}

Node positivity is seen in up to $23 \%$ of patients with positive central compartment lymph nodes who undergo prophylactic level III and IV lateral neck dissection (29). Furthermore, the 5 year-recurrence rate of lateral neck nodes following total thyroidectomy and PCCND was 6\% (30). Lateral node dissection may help in better staging in approximately half of the patients with positive central neck nodes who are at risk of developing lateral neck lymph node involvement. However, there is a lack of evidence to show an improvement in the loco-regional control and overall survival at the expense of overtreatment in more than $75 \%$ of patients. Therefore, prophylactic lateral neck dissection in those without central compartment lymph node involvement is unwarranted $(5,31)$. Personalized decision making is recommended to decide on prophylactic lateral neck dissection in those with central compartment lymph node metastasis, as clear evidence for improvement in outcomes is still not established (5).

\section{Therapeutic lymph node dissection}

Overt metastatic disease in the lateral lymph nodes has been shown to have clinical or radiological central lymph node involvement in $>80 \%$. In those with no evidence of central neck nodes, there is still a high risk of histological involvement (>80\%) (5). Therefore, in patients with overt metastasis in the lateral neck nodes, a therapeutic central and selective lateral neck dissection including levels IIa-Vb lymph nodes is warranted, preserving key structures including the internal jugular vein, accessory nerve and sternomastoid muscle. Therapeutic compartment oriented neck dissection is associated with improved survival and lower recurrence rates and therefore, widely accepted (32). Those with suspicious lateral neck nodes should be confirmed by fine needle cytology before surgery or intra-operative frozen section.

\section{Newer surgical techniques for papillary thyroid cancer}

Newer surgical techniques such as endoscopic thyroidectomy have been utilised to achieve complete resection while improving cosmetic results. Various endoscopic techniques such as axillary, breast, submental and transoral approaches have been described. Despite the experience gained using such techniques in thyroid surgery, the utility in PTC remains controversial. A systematic review and meta-analysis by Chen et al (33) showed that there are limitations in endoscopic surgery due to the difficulty in obtaining views in the restricted space. Furthermore, endoscopic techniques were inferior in terms of operative time, hospital stay and transient recurrent laryngeal nerve palsy. Moreover, despite similar tumour recurrence rates in the short and medium-term, the surgical completeness which may not be optimal could potentially lead to higher longer-term recurrences (33).

\section{Monitoring response to treatment and follow-up}

Post-operative staging of PTC is recommended as a prognostic measure. This provides information to plan for further strategies in relation to surveillance and to decide on therapeutic measures. American Thyroid Association guidelines classify patients into low, intermediate and highrisk categories. This classification depends on the extent of locoregional tumour invasion, adequacy of resection, the aggressiveness of histology, extent of ablation by radioiodine and the presence of specific genetic mutations (e.g.: BRAF). Serum thyroglobulin measurement should be carried out after 6 weeks following total thyroidectomy or radioiodine 
treatment $(34,35)$. TSH suppression in patients who did not receive radioiodine ablation is not recommended and usually maintained at a range between 0.3 to $2.0 \mathrm{mU} / 1$. Patients who underwent total (or near-total) thyroidectomy followed by radioiodine ablation need re-classification based on the risk of cancer recurrence.

This dynamic risk stratification has been shown to correlate closely with the future risk of disease recurrence. For dynamic risk stratification, structural and functional status is obtained by biochemical investigations and imaging after 9 to 12 months of initial treatment with total or hemithyroidectomy and radioiodine ablation. Four types of responses are described according to imaging, biochemical and structural evidence of disease, and used to describe the status of disease during follow-up $(36,37)$.

The excellent response is defined as the absence of radiological, biochemical or structural disease. In these patients, target serum TSH should be in the same range as for the patients who did not undergo radioiodine treatment (36, 38). Patients are categorized as a biochemically incomplete response following the detection of abnormal thyroglobulin levels or rising anti-thyroglobulin antibody levels without any evidence of localized disease.

In the presence of persistent or new loco-regional recurrence or distant metastasis, patients are classified into structurally incomplete response group. In these patients, serum TSH should be maintained below $0.1 \mathrm{mU} / 1$ (36, 38, 39). The indeterminate response is defined when the patient does not show definite biochemical or structural abnormalities that can be classified into benign or malignant categories. In such patients, TSH should be maintained between 0.1 to $0.5 \mathrm{mU} / 1$ for 5 to 10 years and re-assessment is recommended for further management $(36,38,39)$.

\section{Conclusion}

The existing evidence on surgical management of PTC and the current consensus have been reviewed and summarized. A definitive correlation between loco-regional recurrence and long-term survival and the extent of thyroidectomy or lymph node removal have not been established through randomized controlled clinical trials. Therefore, a single surgical strategy may not be universally applicable to all patients. Decision making on the extent of thyroid resection and lymph node dissection should be made by careful analysis of preoperative and intra-operative prognostic factors. This should also be balanced against the risk of surgical morbidity and patient preference. Furthermore, post-operative staging and dynamic risk stratification are important in determining adjuvant therapy and a follow-up plan.
All authors disclose no conflict of interest. The study was conducted in accordance with the ethical standards of the relevant institutional or national ethics committee and the Helsinki Declaration of 1975, as revised in 2000 .

\section{References}

1. Rahib L, Smith BD, Aizenberg R, Rosenzweig AB, Fleshman JM, Matrisian LM. Projecting cancer incidence and deaths to 2030: the unexpected burden of thyroid, liver, and pancreas cancers in the United States. Cancer research. 2014;74(11):2913-21. https://doi.org/10.1158/0008-5472.CAN-14-0155

2. Jayarajah U, Fernando A, Prabashani S, Fernando EA, Seneviratne SA. Incidence and histological patterns of thyroid cancer in Sri Lanka 2001-2010: an analysis of national cancer registry data. BMC cancer. 2018;18(1):163. https://doi.org/10.1186/s12885-018-4083-5

3. Ferlay J, Soerjomataram I, Dikshit R, Eser S, Mathers C, Rebelo $\mathrm{M}$, et al. Cancer incidence and mortality worldwide: sources, methods and major patterns in GLOBOCAN 2012. International journal of cancer. 2015;136(5):E359-E86. https://doi.org/10.1002/ijc.29210

4. Udelsman R, Lakatos E, Ladenson P. Optimal surgery for papillary thyroid carcinoma. World journal of surgery. 1996;20(1):88-93. https://doi.org/10.1007/s002689900016

5. Perros P, Boelaert K, Colley S, Evans C, Evans RM, Gerrard BA $\mathrm{G}$, et al. Guidelines for the management of thyroid cancer. Clinical endocrinology. 2014;81:1-122. https://doi.org/10.1111/cen.12515

6. Cooper DS, Doherty GM, Haugen BR, Kloos RT, Lee SL, Mandel SJ, et al. Revised American Thyroid Association management guidelines for patients with thyroid nodules and differentiated thyroid cancer: the American Thyroid Association (ATA) guidelines taskforce on thyroid nodules and differentiated thyroid cancer. Thyroid. 2009;19(11):1167-214. https://doi.org/10.1089/thy.2009.0110

7. Association BT, editor Guidelines for the management of thyroid cancer2007: Royal College of Physicians.

8. Vasileiadis I, Karakostas E, Charitoudis G, Stavrianaki A, Kapetanakis S, Kouraklis G, et al. Papillary thyroid microcarcinoma: clinicopathological characteristics and implications for treatment in 276 patients. European journal of clinical investigation. 2012;42(6):657-64. https://doi.org/10.1111/j.1365-2362.2011.02633.x

9. Hay ID, Grant CS, Bergstralh EJ, Thompson GB, van Heerden JA, Goellner JR. Unilateral total lobectomy: is it sufficient surgical treatment for patients with AMES low-risk papillary thyroid carcinoma? Surgery. 1998;124(6):958-66. https://doi.org/10.1016/S0039-6060(98)70035-2

10. Mazzaferri EL, Kloos RT. Current approaches to primary therapy for papillary and follicular thyroid cancer. The Journal of Clinical Endocrinology \& Metabolism. 2001;86(4):1447-63. https://doi.org/10.1210/jcem.86.4.7407

11. Nixon IJ, Ganly I, Patel SG, Palmer FL, Whitcher MM, Tuttle RM, et al. Thyroid lobectomy for treatment of well differentiated intrathyroid malignancy. Surgery. 2012;151(4):571-9. https://doi.org/10.1016/j.surg.2011.08.016 
12. Marqusee E, Benson CB, Frates MC, Doubilet PM, Larsen PR, Cibas ES, et al. Usefulness of ultrasonography in the management of nodular thyroid disease. Annals of Internal Medicine. 2000;133(9):696-700.

https://doi.org/10.7326/0003-4819-133-9-200011070-00011

13. Bilimoria KY, Bentrem DJ, Ko CY, Stewart A K, Winchester DP Talamonti MS, et al. Extent of surgery affects survival for papillary thyroid cancer. Annals of surgery. 2007;246(3):375. https://doi.org/10.1097/SLA.0b013e31814697d9

14. Adam MA, Pura J, Gu L, Dinan MA, Tyler DS, Reed SD, et al. Extent of surgery for papillary thyroid cancer is not associated with survival: an analysis of 61,775 patients. Annals of surgery. 2014;260(4):601. https://doi.org/10.1097/SLA.0000000000000925

15. Haigh PI, Urbach DR, Rotstein LE. Extent of thyroidectomy is not a major determinant of survival in low-or high-risk papillary thyroid cancer. Annals of surgical oncology. 2005;12(1):81-9. https://doi.org/10.1007/s10434-004-1165-1

16. Barney BM, Hitchcock YJ, Sharma P, Shrieve DC, Tward JD. Overall and cause-specific survival for patients undergoing lobectomy, near-total, or total thyroidectomy for differentiated thyroid cancer. Head \& neck. 2011;33(5):645-9.

https://doi.org/10.1002/hed.21504

17. Hirsch D, Levy S, Tsvetov G, Shimon I, Benbassat C. Total versus hemithyroidectomy for small unilateral papillary thyroid carcinoma. Oncology letters. 2014;7(3):849-53. https://doi.org/10.3892/ol.2013.1765

18. Chandrasekhar SS, Randolph GW, Seidman MD, Rosenfeld M, Angelos P, Barkmeier-Kraemer J, et al. Clinical practice guideline: improving voice outcomes after thyroid surgery. Otolaryngology-Head and Neck Surgery. 2013;148 (6_suppl):S1-S37). https://doi.org/10.1177/0194599813487301

19. Hermann M, Alk G, Roka R, Glaser K, Freissmuth M. Laryngeal recurrent nerve injury in surgery for benign thyroid diseases: effect of nerve dissection and impact of individual surgeon in more than 27,000 nerves at risk. Annals of surgery. 2002;235(2):261. https://doi.org/10.1097/00000658-200202000-00015

20. Pisanu A, Porceddu G, Podda M, Cois A, Uccheddu A. Systematic review with meta-analysis of studies comparing intraoperative neuromonitoring of recurrent laryngeal nerves versus visualization alone during thyroidectomy. journal of surgical research. 2014;188(1):152-61. https://doi.org/10.1016/j.jss.2013.12.022

21. Randolph G, Clark O. Principles in thyroid surgery. Surgery of the Thyroid and Parathyroid Glands 2nd edition Elsevier, Philadelphia, PA. 2013:273-93.

22. Lorente-Poch L, Sancho J, Ruiz S, Sitges-Serra A. Importance of in situ preservation of parathyroid glands during total thyroidectomy. British Journal of Surgery. 2015;102(4):35967. https://doi.org/10.1002/bjs. 9676

23. Zaydfudim V, Feurer ID, Griffin MR, Phay JE. The impact of lymph node involvement on survival in patients with papillary and follicular thyroid carcinoma. Surgery. 2008;144(6):1070-8. https://doi.org/10.1016/j.surg.2008.08.034
24. Wang TS, Evans DB, Fareau GG, Carroll T, Yen TW. Effect of prophylactic central compartment neck dissection on serum thyroglobulin and recommendations for adjuvant radioactive iodine in patients with differentiated thyroid cancer. Annals of surgical oncology. 2012;19(13):4217-22.

https://doi.org/10.1245/s10434-012-2594-x

25. Randolph GW, Duh Q-Y, Heller KS, LiVolsi VA, Mandel SJ, Steward DL, et al. The prognostic significance of nodal metastases from papillary thyroid carcinoma can be stratified based on the size and number of metastatic lymph nodes, as well as the presence of extranodal extension. Thyroid. 2012;22(11):1144-52.

https://doi.org/10.1089/thy.2012.0043

26. Lang BH-H, Ng S-H, Lau LL, Cowling BJ, Wong KP, Wan KY. A systematic review and meta-analysis of prophylactic central neck dissection on short-term locoregional recurrence in papillary thyroid carcinoma after total thyroidectomy. Thyroid. 2013;23(9):1087-98. https://doi.org/10.1089/thy.2012.0608

27. Wang T S, Cheung K, Farrokhyar F, Roman S A, Sosa J A. A meta-analysis of the effect of prophylactic central compartment neck dissection on locoregional recurrence rates in patients with papillary thyroid cancer. Annals of surgical oncology. 2013;20(11):3477-83 https://doi.org/10.1245/s10434-013-3125-0

28. Hennessy M, Goldenberg D. The Role of Prophylactic Central Neck Dissection in the Treatment of Differentiated Thyroid Cancer. Rambam Maimonides medical journal. 2016;7(1):e0007. https://doi.org/10.5041/RMMJ.10234

29. Ducoudray R, Trésallet C, Godiris-Petit G, Tissier F, Leenhardt L, Menegaux F. Prophylactic lymph node dissection in papillary thyroid carcinoma: is there a place for lateral neck dissection? World journal of surgery. 2013;37(7):1584-91. https://doi.org/10.1007/s00268-013-2020-

30. Barczyński M, Konturek A, Stopa M, Nowak W. Nodal recurrence in the lateral neck after total thyroidectomy with prophylactic central neck dissection for papillary thyroid cancer. Langenbeck's archives of surgery. 2014;399(2):237-44. https://doi.org/10.1007/s00423-013-1135-9

31. Stack B C, Ferris R L, Goldenberg D, Haymart M, Shaha A, Sheth S, et al. American Thyroid Association consensus review and statement regarding the anatomy, terminology, and rationale for lateral neck dissection in differentiated thyroid cancer. 2012. https://doi.org/10.1089/thy.2011-0312

32. Miccoli P, Bakkar S. Surgical management of papillary thyroid carcinoma: an overview. Updates in surgery. 2017;69(2):14550. https://doi.org/10.1007/s13304-017-0449-5

33. Chen C, Huang S, Huang A, Jia Y, Wang J, Mao M, et al. Total endoscopic thyroidectomy versus conventional open thyroidectomy in thyroid cancer: a systematic review and metaanalysis. Ther Clin Risk Manag. 2018;14:2349-61. https://doi.org/10.2147/TCRM.S183612

34. Feldt-Rasmussen U, Petersen P, Date J, Madsen C. Serum thyroglobulin in patients undergoing subtotal thyroidectomy for toxic and nontoxic goiter. Journal of endocrinological investigation. 1982;5(3):161-4. https://doi.org/10.1007/BF03349472 
35. Hocevar M, Auersperg M, Stanovnik L. The dynamics of serum thyroglobulin elimination from the body after thyroid surgery. European journal of surgical oncology. 1997;23(3):208-10. https://doi.org/10.1016/S0748-7983(97)92292-7

36. Tuttle RM, Tala H, Shah J, Leboeuf R, Ghossein R, Gonen M, et al. Estimating risk of recurrence in differentiated thyroid cancer after total thyroidectomy and radioactive iodine remnant ablation: using response to therapy variables to modify the initial risk estimates predicted by the new American Thyroid Association staging system. Thyroid. 2010;20(12):1341-9. https://doi.org/10.1089/thy.2010.0178

37. Vaisman F, Shaha A, Fish S, Michael Tuttle R. Initial therapy with either thyroid lobectomy or total thyroidectomy without radioactive iodine remnant ablation is associated with very low rates of structural disease recurrence in properly selected patients with differentiated thyroid cancer. Clinical endocrinology. 2011;75(1):112-9. https://doi.org/10.1111/j.1365-2265.2011.04002.x

38. Jonklaas J, Sarlis NJ, Litofsky D, Ain KB, Bigos ST, Brierley JD, et al. Outcomes of patients with differentiated thyroid carcinoma following initial therapy. Thyroid. 2006;16(12):1229-42. https://doi.org/10.1089/thy.2006.16.1229

39. Pacini F, Castagna M, Brilli L, Pentheroudakis G, Group EGW. Thyroid cancer: ESMO Clinical Practice Guidelines for diagnosis, treatment and follow-up. Annals of Oncology. 2012;23(suppl_7):vii110-vii9.

https://doi.org/10.1093/annonc/mds230 